TAUP-2480-98

August 6, 2018

\title{
Comments on (Non-)Chiral Gauge Theories and Type IIB Branes
}

\author{
Adi Armonif and Andreas Brandhuber? \\ School of Physics and Astronomy \\ Beverly and Raymond Sackler Faculty of Exact Sciences \\ Tel Aviv University, Ramat Aviv, 69978, Israel
}

\begin{abstract}
We use type IIB brane configurations which were recently suggested by Hanany and Zaffaroni to study four dimensional $\mathrm{N}=1$ supersymmetric gauge theories. We calculate the one loop beta function and realize Seiberg's duality using a particular configuration. We also comment on the anomaly cancelation condition in the case of chiral theories and the beta function in the case of chiral and SO/Sp theories.
\end{abstract}

\footnotetext{
${ }^{1}$ armoni@post.tau.ac.il

${ }^{2}$ andreasb@post.tau.ac.il
} 


\section{Introduction}

In the last two years we have learned that branes are a useful tool in the study of supersymmetric gauge theories [1] (for a recent review and a complete list of references see [2] ). Branes give a nice and effective pictorial meaning to symmetries and parameters of the low energy field theory.

Recently, there has been progress in the realization of chiral theories using branes [3, 4, 5, 6] of type II string theory. In particular, Hanany and Zaffaroni [6] constructed various classical $\mathrm{N}=1$ chiral four dimensional field theories, using brane configurations of type IIB string theory, in the limit of zero string coupling.

In this note we use the suggested construction in the presence of nonzero string coupling. In this case the fivebranes will bend, with asymptotic bending which is dictated by supersymmetry [7, 8]. The bending of a $(p, q)$ fivebrane which is stretched in a line in the $(\mathrm{x}, \mathrm{y})$ plane is

$$
\Delta x: \Delta y=p+q \tau
$$

where $\tau=\frac{i}{g_{s}}+\frac{\chi}{2 \pi}$ and $g_{s}, \chi$ are the string coupling and type IIB axion. Moreover, it was explained in ref. [9] that the slope of each brane should remain constant along the line even if the brane crosses other fivebranes. This constraint leads to anomaly cancelation of local symmetries in the field theory.

The paper is organized as follows: In section 2 we use the bending of the branes to calculate the beta function of $\mathrm{N}=1$ supersymmetric QCD. In section 3 we suggest a brane configuration and brane moves which reproduce Seiberg's duality. In section 4 we introduce a configuration for chiral gauge theories which is determined by anomaly cancelation in the field theory and bending constraints similar to those in [9]. We also give an approximate calculation of the beta function in these cases. We comment on the construction of brane configurations with symplectic and orthogonal groups in section 5 .

Some of the issues that we discuss were previously addressed in the framework of type IIA string theory and M-theory [10, 11, 12, 13, 14]. The issue of finite gauge theories, using type IIB branes was discussed recently in [15]. 


\section{$2 \quad \mathrm{~N}=1 \mathrm{SQCD}$ setup and the beta function}

The brane configuration that we use is similar to the one that was introduced in ref. [6]. The configuration (Fig. 1) consists of two horizontal lines which represent two NS branes with world volume 012367 (which we will denote by NS') and two vertical lines which represent NS branes with world volume 012345 (which we will denote by NS). In addition, there are D5 brane with world volume 012346 which are attached to the NS and NS' branes. This set of branes leads to $N=1$ SQCD in four dimensions with generically chiral matter. The numbers in the boxes of Fig.1 correspond to the number of D5 branes. The central D5 branes are finite in two directions and represent gauge symmetries in the four dimensional gauge theory. Semi-infinite boxes represent global symmetries. The intersection of the NS and NS' brane might lead to additional light states, e.g. D1 strings connecting NS and NS'. These states might affect the 4D field theory, but we believe that the results which are presented in this paper are not influenced by them. The direction of allowed arrows are North, East and South-West [6]. Outgoing arrows are fundamentals and in-going arrows are anti-fundamentals of the gauge group. Thus Fig.1 represents four dimensional $N=1$ supersymmetric $S U(N)$ with $N_{c}+X+Y$ fundamentals and $N_{c}+X+Y$ anti-fundamentals. Notify that the global symmetry factories into a left and right part. This is to be contrasted to other type IIA constructions of N=1 SQCD where only the diagonal subgroup of the global flavor symmetry is manifest. Note also that in this construction $N_{f} \geq N_{c}$ (since $X$ and $Y$ cannot be negative). 


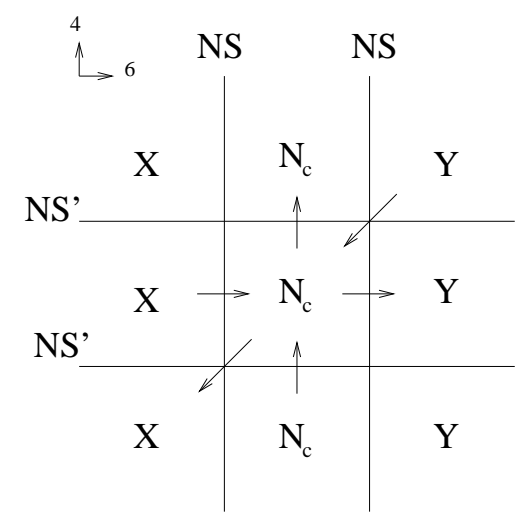

Figure 1: $N=1$ Theory with $N_{f}=N_{c}+X+Y$ flavors

The construction guarantees that the bending of the four NS branes is constant along each brane. This is a necessary condition to ensure that the gauge symmetry is anomaly free [9]. In addition, note that the NS' branes have zero bending, since we put the same number of D5 branes on each side of them.

The coupling constant of the gauge theory is related to the area of the middle box.

$$
\frac{1}{g^{2}}=\frac{\Delta x_{4} \Delta x_{6}}{g_{s} l_{s}^{2}}
$$

The running of the coupling constant is due to the bending of the branes that surround the middle box. In the type IIA construction of $N=1$ gauge theories, one could not define a distance which is directly related to the running coupling because the D4 branes end on branes whose world-volume extends in different directions. However, since in our construction the two NS' branes do not bend and their distance is constant, we can relate the beta function to the change of the distance between the two NS branes. The result is

$$
b_{0}=\left(X-N_{c}\right)+\left(Y-N_{c}\right)=N_{f}-3 N_{c},
$$

which agrees with the field theory result! 


\section{$3 \quad \mathrm{~N}=1$ Duality}

In this section we would like to propose a brane motion that can be related to Seiberg's duality [16]. The realization of $N=1$ Duality using branes in type IIA string theory was first demonstrated in [10, 11]. Here we show that a simple construction in type IIB leads to this duality, too.

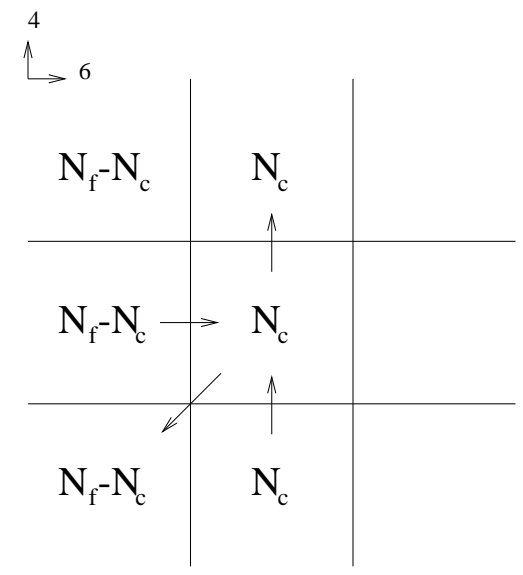

Figure 2: The "electric" theory. $S U\left(N_{c}\right)$ gauge theory with $N_{f}$ flavors

Consider the theory in Fig.2 . This theory describes $N=1$ supersymmetric $S U\left(N_{c}\right)$ gauge theory with $N_{f}$ fundamentals and $N_{f}$ anti-fundamentals. We refer to this theory as the "electric" theory. Now attach an additional NS brane to the D5 branes at $x_{6}=-\infty$. As long as the additional NS brane is positioned at infinity the theory on the branes remains unchanged. Now we move the NS brane to a finite $x_{6}$ position. In addition, take the right NS brane and bring it to $x_{6}=\infty$. The resulting theory is described in Fig. 3. This theory is an $S U\left(N_{c}\right)$ theory with $N_{f}-N_{c}$ flavors. We refer to this theory as the "magnetic" theory. 


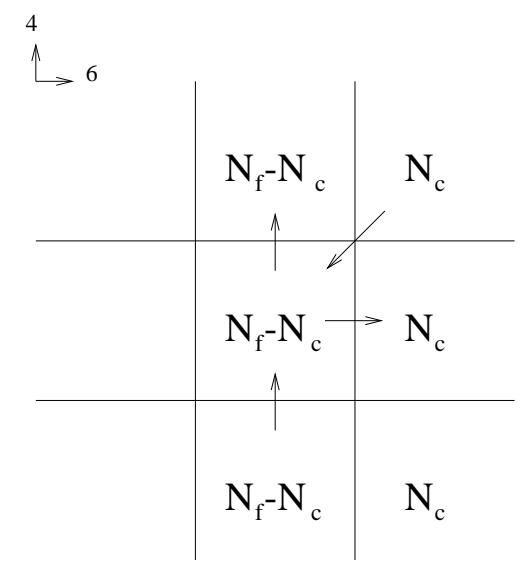

Figure 3: The "magnetic" theory. $S U\left(N_{f}-N_{c}\right)$ gauge theory with $N_{f}$ flavors

The intermediate theory (the left and the right NS are at finite distance in the $x_{6}$ position) is not dual to either the electric or the magnetic theories. In fact this is a theory with an $S U\left(N_{c}\right) \times S U\left(N_{f}-N_{c}\right)$ gauge group and the following matter content

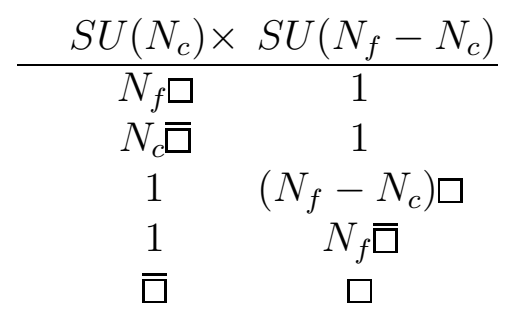

Table 1: The matter content of the 'intermediate' theory

However, the magnetic and the electric theories can be viewed as two limits of this intermediate theory.

While we cannot justify the motion of the branes which leads to the duality, it reproduces the known field theory results.

As a simple test of the above suggestion, we will prove that the relation between giving a mass terms in the electric theory and Higgsing in the mag- 
netic theory (and vice versa) holds in the brane configuration. Adding a mass term for one of the quarks in the electric theory reduces the flavor symmetry by one. In the magnetic theory this corresponds to giving a mass to one of the mesons which via the equations of motion induces a vev for one of the magnetic quarks reducing both colors and flavors by one unit.

This correspondence can be seen in the brane construction in the following way: reconnect the D5 brane in the left-upper box of Fig. 2 to the two other D5 branes in the left column. Now the D5 brane is infinite in the $x_{4}$ direction and can be lifted in the $x_{5}$ direction. The $x_{5}$ position is proportional to the mass term. The result is an electric theory with $N_{f}-1$ flavors. Introducing the additional NS brane and moving the right NS brane, we obtain the magnetic theory in which the middle column of Fig.3 has $N_{f}-N_{c}-1$ D5 branes. The $x_{5}$ position is related to the mass term for the meson. This magnetic theory has $N_{f}-N_{c}-1$ colors and $N_{f}-1$ flavors, as expected.

In the same way we can show the relation between Higgsing in the electric theory and mass terms in the magnetic theory. In this case we reconnect D5 branes in the middle column of fig. 2 to obtain one D5 brane that is infinite in the $x_{4}$ direction but finite in the $x_{6}$ direction and lift it in the $x_{5}$ direction. This reduces color and flavor by one and corresponds to Higgsing in the electric theory where the $x_{5}$ position is proportional to $\langle Q \tilde{Q}\rangle$. After the brane motion we obtain the magnetic theory with flavor reduced by one which is related to giving a mass term to one of the quarks in the magnetic theory.

In the above discussion, we did not consider the superpotential in the two dual descriptions. Duality of the field theories requires zero superpotential in the electric theory and a superpotential of the form $W=M q \tilde{q}$ in the magnetic theory. Since in our description the flavor symmetry boxes are semi-infinite, the mesons in both sides are not dynamical, in contrast to the situation in the magnetic theory in field-theory. This obstacle can probably be solved by adding an extra NS brane at large value of $x_{6}$ in the magnetic brane configuration. We leave this issue as an open question.

From the field theory point of view it looks like two different degenerations of a field theory with a product gauge group. By decoupling one of the factors we turned a local symmetry into global flavor symmetry. One also would like to check that the moduli spaces in the electric and magnetic theory match. We have given some evidence for this by relating massterms and Higgsing in the dual theories. Maybe the introduction of D7 branes is necessary (similar 
to the use of D6 branes in type IIA) to introduce matter to make this match complete. In the type IIA brane construction of Seiberg's duality [10] a certain brane creation process was a crucial ingredient. In this approach the moduli space could be matched precisely and the meson was identified. But this approach also has disadvantages e.g. only a diagonal subgroup of the flavor symmetry is manifest and an Fayet-Ilioupoulos term had to be introduced to avoid singularities in the brane motion. Such a singularity was never a problem the type IIB approach. In summary it must be said that any brane construction of Seiberg's duality presented so far captures many features of the field theory duality but has also some problems.

\section{Anomaly cancelation in chiral theories}

In this section we study $S U\left(N_{c}\right)$ gauge theories with an symmetric/antisymmetric tensor, $N_{f}$ fundamentals and $\bar{N}_{f}$ anti-fundamentals. Anomaly cancelation requires $\bar{N}_{f}-N_{f}=N_{c}+4$ (or $\bar{N}_{f}-N_{f}=N_{c}-4$ ) for theories including the symmetric (or anti-symmetric) representation.

We cannot derive this result from string theory but the configuration is fixed by the requirement that NS branes have constant bending and that the field theory anomaly is canceled. The relevant brane configuration for the symmetric case is described in Fig.4 


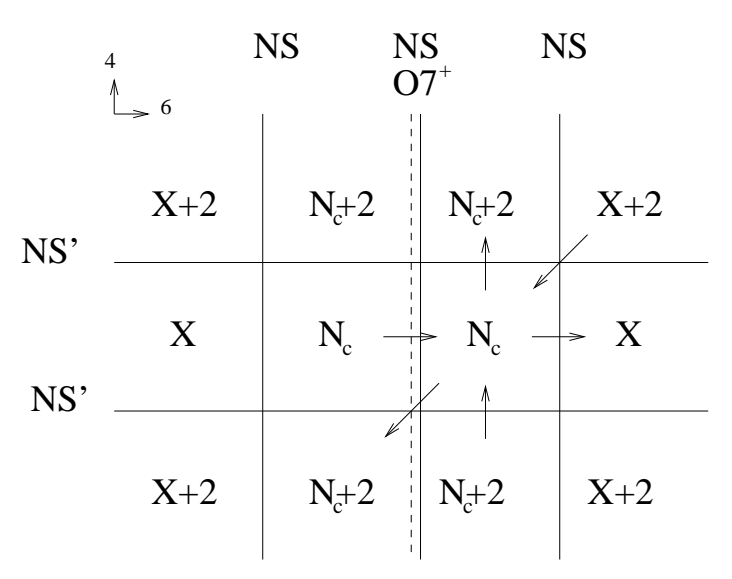

Figure 4: $N=1 S U(N)$ theory with symmetric, fundamentals and antifundamentals. The dashed line denote the $\mathrm{O}^{+}$.

In addition to the three NS branes, the two NS' branes and the D5 branes we added on top of the middle NS brane an orientifold-sevenplane with world volume 01234789 . The presence of the orientifold induces a $Z_{2}$ symmetry with respect to the $x_{5}$ and $x_{6}$ directions. An orientifold plane with RR charge -8 $(+8)$ will be denoted by $O 7^{-}\left(O 7^{+}\right)$and gives rise to a $S U(N)$ gauge theory with an anti-symmetric (symmetric) representation.

In order to ensure that the bending of each NS brane remains constant along the brane, we added additional D5 branes around the $N_{c}$ D5 branes in the middle. The additional branes are responsible for extra matter in the fundamental and anti-fundamental of the gauge group. Above the upper NS' brane there should be 2 extra D5 brane at each side of the mirror (and similarly two extra D5 branes below the lower NS', at each side), to cancel the chiral anomaly in field theory. Although we have no string theory argument for this rule, it should be possible to derive it directly from string theory. Note that it is not sufficient to require a constant bending. There should be two extra D5 branes above and below the middle row. Any other number of D5 branes leads to an anomaly in the field theory. We assume that this effect is due to the presence of the orientifold plane.

Let us count the number of fundamentals/anti-fundamentals which interact with the color group. The in-going arrows are anti-fundamentals and the outgoing arrows are fundamentals. There is one exception: The South- 
West arrow which crosses the $\mathrm{O}^{+}$plane is counted as anti-fundamental and not as fundamental. The argument for this is the following: A superpotential term $A \tilde{Q} \tilde{Q}$ is formed by the symmetric (the horizontal arrow which connect the middle box to its mirror), the anti-fundamentals (the vertical arrow which connects the lower box to the middle box in the mirror) and the South-West arrow. Since the superpotential is a scalar, the diagonal arrow should represent anti-fundamentals. Hence we have $\bar{N}_{f}=2 N_{c}+X+6$ and $N_{f}=N_{c}+X+2$.

We conclude that

$$
\bar{N}_{f}-N_{f}=N_{c}+4 .
$$

Similarly, in the case of anti-symmetric matter and $O 7$ plane we obtain

$$
\bar{N}_{f}-N_{f}=N_{c}-4
$$

We can also calculate, approximately, the beta function of these theories. As we explained in section 2, the relation between the one-loop beta function and the bending of the branes, is meaningful only when two of the branes that surround the gauge group box remain parallel. In the current configuration this is not the case, since the two NS' branes are not parallel. The D5 and the $\mathrm{O}^{+}$bend the NS' brane in different directions and therefore the NS' can never be made flat in all directions. However, if the bending of the right NS brane is much larger than the bending of the NS' brane, we expect to get an approximate answer. By approximate we mean that the dependence on $N_{c}$ and $N_{f}$ is correct.

The bending of the right NS is $X-N_{c}$, hence

$$
b_{0} \approx N_{f}-2 N_{c}-2
$$

Note that the field theory result is

$$
b_{0}=N_{f}-2 N_{c}+3
$$

Which indeed agrees when $2 N_{c}-N_{f} \gg 1$. Similarly, the approximate value of the beta function can be calculated in the case of the chiral theory with anti-symmetric matter. 


\section{$5 \quad$ SO and Sp Gauge Groups}

We would like to comment on the one-loop beta function in the case of $N=1$ SQCD with gauge groups $S O\left(N_{c}\right)$ and $S p\left(N_{c}\right)$ (with $N_{c}$ even). The realization of these models was worked out in [6] and it is similar to the theory in Fig.4. The brane configuration for $S O\left(N_{c}\right)$ is given in Fig.5

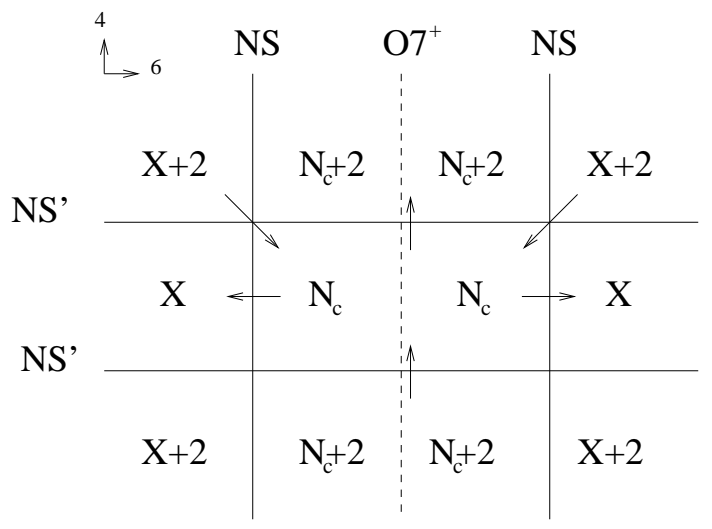

Figure 5: $N=1 S O\left(N_{c}\right)$ theory. The dashed line denote the $O 7^{+}$.

Note that in contrast to the previous case, there is no NS brane at $x_{6}=0$ and the color box should be understood as one box. Here we assume that the orientifold plane imposes the same constraints on the number of branes in the upper and lower row as in the previous section. This brane rule can be supported by field theory arguments. Chiral theories and orthogonal/symplectic theory are closely related by duality[17]. A theory with $S p\left(N_{c}-4\right) \times S U\left(N_{c}\right)$ gauge group can be constructed in a similar way to the theory in Fig.5, but with an $O 7$ plane and additional NS branes at finite $x_{6}$ and $-x_{6}$. By moving the middle NS brane and its mirror towards the orientifold plane and moving one of them to $x_{7}=\infty$ we obtain an $S U\left(N_{c}\right)$ theory with antisymmetric tensor and extra matter. This process was already described in ref.[6]. Here we conclude that two extra D5 branes above and below the gauge box in the chiral case (Fig.4), are also necessary in the orthogonal and symplectic cases.

According to the rules of the previous section, we will not be able to get the accurate beta function of these theories since a completely flat NS' brane 
cannot be achieved. But it is again possible to get the correct dependence on $N_{f}$ and $N_{c}$. The number of flavors in this case is

$$
2 N_{f}=4 X+2 N_{c}+8
$$

The one-loop beta function is obtained, approximately, by $2 X-2 N_{c}$

$$
b_{0} \approx N_{f}-3 N_{c}-4
$$

in agreement with the field theory result

$$
b_{0}=N_{f}-3 N_{c}+6 .
$$

A similar result can be obtained for the $S p$ case. In this case we replace the $O 7^{+}$by $O 7$, and we again capture the $N_{f}-3 N_{c}$ dependence.

\section{Acknowledgments}

We thank Nissan Itzhaki, Udi Fuchs and especially Shimon Yankielowicz for discussions.

\section{References}

[1] A. Hanany and E. Witten, "Type IIB Superstrings, BPS Monopoles, and Three-Dimensional Gauge Dynamics", Nucl. Phys. B492 (1997) 152, hep-th/9611230.

[2] A. Giveon and D. Kutasov, "Brane Dynamics and Gauge Theory", hepth/9802067.

[3] K. Landsteiner, E. Lopez and D.A. Lowe, "Duality of Chiral N=1 Supersymmetric Gauge Theories via Branes", hep-th/9801002.

[4] I. Brunner, A. Hanany, A. Karch and D. Lüst, "Brane Dynamics and Chiral non-Chiral Transitions", hep-th/9801017.

[5] S. Elitzur, A. Giveon, D. Kutasov and D. Tsabar, "Branes, Orientifolds and Chiral Gauge Theories", hep-th/9801020. 
[6] A. Hanany and A. Zaffaroni, "On the Realization of Chiral FourDimensional Gauge Theories Using Branes", hep-th/9801134.

[7] O. Aharony and A. Hanany, "Branes, Superpotentials and Superconformal Fixed points",Nucl. Phys. B504 (1997) 239, hep-th/9704170.

[8] O. Aharony, A. Hanany and B. Kol, "Webs of $(p, q)$ 5-branes, Five Dimensional Field Theories and Grid Diagrams", hep-th/9710116.

[9] E. G. Gimon and M. Gremm, "A note on brane boxes at finite string coupling", hep-th/9803033.

[10] S. Elitzur, A. Giveon and D. Kutasov, "Branes and N=1 Duality in String Theory", Phys. Lett. 400B (1997) 269, hep-th/9702014.

[11] S. Elitzur, A. Giveon, D. Kutasov, E. Rabinovici and A. Schwimmer, "Brane Dynamics and N=1 Supersymmetric Gauge Theory", Nucl. Phys. B505 (1997) 202, hep-th/9704104.

[12] K. Hori, H. Ooguri and Y. Oz, "Strong Coupling Dynamics of Four Dimensional N=1 Gauge Theories from M Theory Fivebrane", hepth/9706082.

[13] E. Witten, "Branes and the Dynamics of QCD", Nucl. Phys. B507 (1997) 658, hep-th/9706109.

[14] A. Brandhuber, N. Itzhaki, V. Kaplunovsky, J. Sonnenschein and S. Yankielowicz, "Comments on the $M$ Theory Approach to $N=1 S Q C D$ and Brane Dynamics", Phys. Lett. 410B (1997) 27, hep-th/9706127.

[15] A. Hanany, M. J. Strassler and A. M. Uranga, "Finite Theories and Marginal Operators on the Brane", hep-th/9803086.

[16] N. Seiberg, "Electric-Magnetic Duality in Supersymmetric Non-Abelian Gauge Theories", Nucl. Phys. B435 (1995)129, hep-th/9411149.

[17] M. Berkooz, "The Dual of Supersymmetric SU $(2 k)$ with an Antisymmetric Tensor and Composite Dualities", Nucl. Phys. B452 (1995) 513, hep-th/9505067. 